\title{
Synthesis and characterization of sensitive hydrogels based on semi-interpenetrated networks of poly[2-ethyl-(2-pyrrolidone) methacrylate] and hyaluronic acid
}

\author{
Joana Magalhães, ${ }^{1}$ Rui A. Sousa, ${ }^{2}$ João F. Mano, ${ }^{2}$ Rui L. Reis, ${ }^{2}$ \\ Francisco J. Blanco, ${ }^{1}$ Julio San Román ${ }^{3}$ \\ ${ }^{1}$ Rheumatology Division, CIBER-BBN/ISCIII, Tissue Engineering and Cellular Therapy Group (CBTTC-CHUAC), INIBIC-Hospital \\ Universitario A Coruña, C/As Xubias, S/N 15006 A Coruña, Spain \\ ${ }^{2} 3 B^{\prime}$ 's Research Group Biomaterials, Biodegradables and Biomimetics, University of Minho, Headquarters of the European \\ Institute of Excellence on Tissue Engineering and Regenerative Medicine, AvePark, 4806-909 Taipas, Guimarães, Portugal \\ ${ }^{3}$ Polymer Science and Technology Institute, CSIC and CIBER-BBN, C/Juan de la Cierva, 3, 28006 Madrid, Spain
}

Received 1 December 2011; revised 15 May 2012; accepted 25 May 2012

Published online 25 July 2012 in Wiley Online Library (wileyonlinelibrary.com). DOI: 10.1002/jbm.a.34312

\begin{abstract}
Sensitive hydrogels attract interest due to their soft wet appearance and shape response to environmental variations. The synthesis and characterization of semi-interpenetrated hydrogels obtained by radical-induced polymerization of 2-ethyl-(2-pyrrolidone)methacrylate (EPM) in the presence of different concentrations of hyaluronic acid (HA) using $\mathrm{N}, \mathrm{N}^{\prime}$-methylene-bisacrylamide or triethylene glycol dimethacrylate as crosslinker, followed by freeze-drying, are described. Polymeric systems were characterized by NMR, FTIR, SEM, TGA, and DMA. PEPMHA hydrogels' mechanical properties and swelling were found to be intimately related to HA concentration and crosslinker. The swelling response was assessed for temperature and $\mathrm{pH}$ variation in order to
\end{abstract}

study the behavior of the hydrogels. We found that the presence of HA in PEPM polymeric systems induced a sensitivity to $\mathrm{pH}$ variation rather than temperature. Finally, the biocompatibility profile of the hydrogels was evaluated, using mesenchymal stem cells. Cell adhesion and proliferation results revealed the non-cytotoxicity of the systems. We estimate that PEPMHA hydrogels can be used for applications in tissue engineering and for the controlled release of bioactive compounds. ( 2012 Wiley Periodicals, Inc. J Biomed Mater Res Part A: 101A: 157-166, 2013.

Key Words: hyaluronic acid, vinyl pyrrolidone, hydrogels, hydrophilic polymers, stimuli-sensitive polymers

How to cite this article: Magalhães J, Sousa RA, Mano JF, Reis RL, Blanco FJ, Román JS. 2013. Synthesis and characterization of sensitive hydrogels based on semi-interpenetrated networks of poly[2-ethyl-(2-pyrrolidone) methacrylate] and hyaluronic acid. J Biomed Mater Res Part A 2013:101A:157-166.

\section{INTRODUCTION}

Hydrogels are crosslinked polymer networks that can swell up to $99 \%$ water, which renders them with better lubricating properties, low coefficients of friction, and high mechanical strength, making them ideal for transmitting loads. ${ }^{1}$ These materials are of especially useful for the controlled release of drugs, and these are currently being used in the field of tissue engineering (TE) as three-dimensional scaffolds, cell delivery agents, and cell carriers. ${ }^{2,3}$

The development of appropriate hydrogel matrices is critical for the success of any approach. If we consider bone and cartilage TE applications, the hydrogel matrix should be composed of biocompatible and biodegradable materials and should exhibit mechanical properties that can sustain load bearing. ${ }^{4-6}$

Hydrogel matrices have been fabricated widely from polymers of both natural and synthetic origin. ${ }^{5}$ Even though naturally derived materials are intrinsically biocompatible and physiologically degradable, several limitations are encountered; for instance, they are generally weak and lack batch-to-batch consistency. ${ }^{7}$ On the other hand, hydrogels based on synthetic polymers that lack cell recognition sites offer a range of easily tailored properties that are advantageous for a reproducible fabrication. ${ }^{8}$ Therefore, several groups have been investigating the utility of biosynthetic composite hydrogels composed of both synthetic and naturally occurring extracellular matrix (ECM) components so as to exploit the advantages of both polymer types. ${ }^{9-11}$

One approach of creating composite hydrogels is the fabrication of an interpenetrating network (IPN) or semi-IPN (sIPN) of polymers. A sIPN consists of a polymer network containing a molecularly entangled second polymer. $^{12}$ Although IPN can achieve significant improvement in strength and stiffness, they are often nondegradable and not

Correspondence to: J. Magalhães; e-mail: joana.cristina.silva.magalhaes@sergas.es

Contract grant sponsor: Marie Curie Early Stage Training Alea Jacta Est; contract grant number: MEST-CT-2004-8104 
applicable for cell culture. Therefore, to develop composite sIPNs, we propose to design a hydrogel matrix using a synthetic origin polymer in the presence of a natural biopolymer.

For this, we have focused on the current applications of poly(vinyl pyrrolidone) (PVP) and its derivatives on both cartilage and bone TE. ${ }^{13}$ Yet, the monomer vinyl pyrrolidone (VP) exhibits poor reactivity in free radical polymerization reactions. It is known that the reactivity of systems based on the pyrrolidone ring can be enhanced by introducing a methacrylate group, which leads to materials with properties that are similar to poly(2-hydroxyethyl methacrylate), and these materials developed in the 1960s have also been considered in numerous biomedical applications due to their high water uptake capacity, soft consistency, and low superficial tension. ${ }^{14}$

Gonzalez et al. described hydrophilic monomers with chemical structures that are related to those of PVP and poly(2-hydroxyethyl methacrylate) by maintaining their biocompatible character but with improved solubility in water. ${ }^{15}$ Ethyl pyrrolidone [2-ethyl-(2-pyrrolidone)methacrylate] (EPM) and ethyl pyrrolidine [2-ethyl-(2-pyrrolidine)methacrylate] (EPyM) originated hydrophilic polymers, which constitute the basis of the production of hydrogels with sensitivity to temperature and/or $\mathrm{pH}$, and both present reversible character. This feature makes them belong to a class of materials designated as stimuli-sensitive or smart hydrogels, which undergo changes on their swelling ratio in response to small variations in environmental conditions, such as temperature, $\mathrm{pH}$, ionic strength, and pressure. ${ }^{16,17}$

Moreover, focusing on sIPNs based on the thermosensitive polymer EPM, we aimed to physically entangle hyaluronic acid (HA), an abundant glycosaminoglycan present as a major constituent of the ECM, which could ultimately facilitate cell recognition and proliferation on these hybrid materials. HA is a ubiquitous molecule but can be mainly found in skin, vitreous eye ball, and cartilage tissue. ${ }^{18}$ Due to its unique viscoelastic properties, it plays an important role in lubrication as a component of the synovial fluid of joints. ${ }^{19}$ In aqueous solutions, the hydrogen bonds established between water molecules and the adjacent carboxyl and $\mathrm{N}$ acetyl groups of the HA are responsible for a conformational stiffness of the polymer, limiting its flexibility. Due to its highly negative charge, HA attracts positive ions, leading to an osmotic imbalance in aqueous solutions. Because of this water-binding retention capability, this biopolymer has proven to be ideal for the preparation of semi-interpenetrating hydrogels. ${ }^{20,21}$

To stabilize the proposed hydrophilic water soluble polymers, we have considered the application of two multifunctional crosslinking agents, $N, N^{\prime}$-methylene-bisacrylamide (MBA) and triethylene glycol dimethacrylate (TEGDMA). These crosslinking agents due to their polar functional groups can increase the hydrophilic nature of the hydrogel and therefore are commonly used for the synthesis of hydrogels with high water retention capacity. ${ }^{22}$ Moreover, MBA presents functional groups in common with the EPM monomer and so will not alter significantly the chemical structure of the ending hydrogels.
In this paper, we describe the preparation and physical or chemical characterization of a hybrid material based on semi-interpenetrating networks obtained by the radically induced polymerization of EPM in the presence of moderate levels of unmodified $\mathrm{HA}$ and two different crosslinking agents, MBA or TEGDMA. The influence of crosslinkers and concentration of HA on the temperature-induced phase transition behavior and swelling characteristics of the hydrogel systems was investigated over a defined range of temperature and $\mathrm{pH}$. To evaluate the in vitro cytocompatibility of the PEPMHA hydrogels, we performed cell viability studies, using mesenchymal stem cells.

\section{MATERIAL AND METHODS}

\section{Synthesis of EPM monomer}

2-ethyl-2-(pyrrolidone)methacrylate (EPM) was synthesized based on a previously described protocol. ${ }^{15}$ Briefly, to a solution of $10 \mathrm{~g}$ of 2-hydroxyethyl-pyrrolidone (EP; 0.0774 $\mathrm{mol})$ in chloroform $(75 \mathrm{~mL})$ with $10.7 \mathrm{~mL}(0.0774 \mathrm{~mol})$ of triethylamine a solution of $0.116 \mathrm{~mol}$ of methacryloyl chloride $(\mathrm{MeCl})$ in chloroform $(25 \mathrm{~mL})$ was added dropwise at $0^{\circ} \mathrm{C}$ under a nitrogen atmosphere with magnetic stirring. The solution was left overnight under these conditions. The obtained solution was washed several times with an aqueous solution of sodium hydroxide $(\mathrm{NaOH}, 5 \% \mathrm{wt})$ and dried over anhydrous sodium sulfate $\left(\mathrm{Na}_{2} \mathrm{SO}_{4}\right)$. The solvent was removed at reduced pressure.

\section{Preparation of sIPNs PEPMHA hydrogels}

Free radical polymerization of EPM $([\mathrm{EPM}]=0.725 \mathrm{M})$ was performed at $50^{\circ} \mathrm{C}$ in the presence of aqueous solutions with different concentrations of low molecular weight HA (LMW, 2.5 and 5\% (w/v), Bioiberica), using MBA or TEGDMA (2.0 and 1.7\% molar fraction, Fluka), as crosslinker and potassium persulfate $\left(\left[\mathrm{K}_{2} \mathrm{~S}_{2} \mathrm{O}_{8}\right]=2 \times 10^{-2} M\right)$, as radical initiator, forming sIPNs. After 4 hours of reaction, the PEPMHA hydrogels formed were demolded and consecutively washed with distilled water. For freeze-drying, samples were placed in a $-20^{\circ} \mathrm{C}$ freezer for 12 hours. The temperature of the freeze-dryer (LyoQuest -80, Telstar) was reduced to $-40^{\circ} \mathrm{C}$ and held at this temperature for 1 hour. The samples were subsequently maintained at $-80^{\circ} \mathrm{C}$ for drying in vacuum (0.030 mbar) for 48 hours. Discs ( $4 \mathrm{~mm}$ diameter $\times 2.5 \mathrm{~mm}$ ) were punched from the lyophilized samples and stored at $5^{\circ} \mathrm{C}$ until use.

\section{Characterization measurements}

Attenuated total reflectance Fourier transform infrared (ATR-FTIR). ATR-FTIR spectroscopy analysis of the PEPMHA hydrogels was performed on a PerkinElmer Spectrum One spectrophotometer operating in ATR mode (ATR- Diamond $\mathrm{Se}-\mathrm{Zn}$ ) in order to confirm the chemical structure of the samples produced.

Thermal analysis. The thermogravimetric analysis of PEPMHA hydrogels was obtained with a Perkin-Elmer TGA7 thermobalance. The experiments were performed under helium flux of $90 \mathrm{~mL} / \mathrm{min}$, at a heating rate of $10^{\circ} \mathrm{C} / \mathrm{min}$ from 0 to $500^{\circ} \mathrm{C}$, using samples with approximately $10 \mathrm{mg}$. 
Scanning electron microscopy. The surface morphology of the freeze-dried hydrogels, previously thinly metal coated $(\mathrm{Au}: \mathrm{Pd} / 80: 20)$, was determined by scanning electron microscopy (SEM) using a Philips XL30-ESEM microscope operated at an accelerating voltage of $15 \mathrm{kV}$. Photographs were taken at high magnifications $(\times 2000)$.

Dynamic mechanical analysis. Dynamic mechanical analysis was performed using a TRITEC2000B DMA (Triton Technology, UK), equipped with a compression mode, with a displacement of $50 \mu \mathrm{m}$, in a controlled environment at $37^{\circ} \mathrm{C}$. PEPMHA-lyophilized samples were rehydrated in distilled water until equilibrium was reached. After that, hydrogels were analyzed in the DMA apparatus, while immersed in a liquid bath placed in a Teflon ${ }^{\circledR}$ reservoir. After equilibration, DMA spectra were obtained during a frequency scan that varied between 0.1 and $10 \mathrm{~Hz}$.

Swelling studies. For swelling experiments, pre-weighed freeze-dried samples were immersed in a phosphate buffer saline solution (PBS; pH 7.4) set in a temperature-controlled bath at $37^{\circ} \mathrm{C}$. At defined time intervals, swollen hydrogels were sampled, excess water was removed with filter paper, and the weight of the samples was determined. In parallel, to determine the temperature- or $\mathrm{pH}$-dependent swelling properties, hydrogels were immersed in PBS at $\mathrm{pH} 2,7.4$, and 10 and exposed to a consecutive increment of temperature $\left(20-50^{\circ} \mathrm{C}\right)$, and the water uptake was monitored over time. The swelling ratio was determined gravimetrically, in triplicate, according to the following equation:

$$
\% S=\frac{W_{t}-W_{0}}{W_{0}} \times 100
$$

where $\% \mathrm{~S}$ is the swelling ratio, $W_{\mathrm{t}}$ and $W_{0}$ are the sample weight on the swollen (at time $t$ ) and lyophilized-dry state, respectively.

\section{Cell culture}

PEPMHA hydrogels prepared with TEGDMA or MBA and HA $5 \%$ were tested. Samples were presterilized under UV light and prehydrated with Dulbecco's modified Eagle medium (DMEM) before cell seeding. Porcine bone marrow mesenchymal stem cells (BM-MSCs) were obtained from femurs of 3-month-old large white healthy pigs (Coren SL, A Coruña, Spain). Isolated bone marrow cells were cultured in monolayer in DMEM supplemented with $20 \%$ fetal bovine serum (FBS), 1\% penicillin and streptomycin (all from Sigma Aldrich, St. Louis, MO, USA) until 90\% confluence. BM-MSCs were then seeded in a 24-well plate containing the test specimens at a density of $5 \times 10^{5}$ cells $/ \mathrm{mL}$ with a total volume of $1 \mathrm{~mL}$ medium within each well (cells only were used as control). After 48 hours, cell viability was assessed by the alamar blue cell proliferation assay. For histological analysis, cell constructs were fixed in formaldehyde, embedded in paraffin, and processed using standard histological procedures. The histological sections (4 $\mu \mathrm{m}$ thick) were stained with hematoxylin and eosin (H\&E).

\section{Statistical analysis}

Statistical analyses were performed using a one-way analysis of variance (ANOVA). Differences were considered statistically significant for $p<0.05$.

\section{RESULTS}

\section{Preparation and FTIR-ATR characterization of PEPMHA}

The methacrylic monomer EPM was synthesized with high yields $(\sim 90 \%)$ by reaction of the hydroxyl group of the EP with methacryloyl chloride, resulting in a methacrylic ester. Figure 1 shows the FTIR-ATR spectra of hyaluronic acid, EP, EPM, and PEPMHA systems, confirming the evolution of the chemical reactions and the formation of a polymeric structure. In the spectra region between 3600 and $2800 \mathrm{~cm}^{-1}$, two broad absorption bands were present that are typically exhibited by polysaccharides: the $\mathrm{O}-\mathrm{H}$ stretching vibration $(\mathrm{vH})$, corresponding to the broad band situated between $3600-3000 \mathrm{~cm}^{-1}$ and the $\mathrm{C}-\mathrm{H}$ stretching vibration $(\mathrm{vCH})$, corresponding to the bands situated between 3000-2800 $\mathrm{cm}^{-1}$. In addition, $\mathrm{N}-\mathrm{H}$ stretching vibrations $(\mathrm{vNH})$ are found to be overlapped with the $0-\mathrm{H}$ band. ${ }^{23} \mathrm{HA}$ spectrum also exhibits several overlapped bands in the region of carbonyl stretching vibrations, with the highest peak at 1608 $\mathrm{cm}^{-1}$ (vCOO-) and in the right side appears the amide II band $\left(1558 \mathrm{~cm}^{-1}\right)$.

In the EP spectrum, the band situated at $1662 \mathrm{~cm}^{-1}$ is assigned to the $v \mathrm{C}=0$ of the amide group present in the pyrrolidone ring. The band at $1600 \mathrm{~cm}^{-1}\left(\mathrm{vCH}_{2}=\mathrm{C}\right)$ corresponding to the double bond is a result of the methacrylation reaction, during EPM formation. PEPMHA spectrum combines the bands associated with HA and EPM. The overlapping of the carbonyl and amide vibrations of both compounds results in a broad band situated between 1800 and $1500 \mathrm{~cm}^{-1}$, with a peak at $1650 \mathrm{~cm}^{-1}$ (vC=0 cycle), a left band at $1723 \mathrm{~cm}^{-1}(\mathrm{vC}=0)$, corresponding to a characteristic ester bond, and on the right a shift is visible, which can be both attributed to the disappearance of the double bond signal present in the EPM spectrum, which gave rise to the polymeric chain and a sign of possible interactions between $\mathrm{HA}$ and PEPM.

\section{Thermal analysis characterization}

The thermal stability of PEPMHA was determined by thermogravimetry. The decomposition profiles and associated first derivative curves of PEPMHA are shown in Figure 2. This figure gives clear evidence that the polymer has great thermal stability up to $240^{\circ} \mathrm{C}$, when weight loss due to degradation begins. Beyond this temperature, the rate of weight loss decreases continuously as indicated in the thermogram by the different decomposition regions. The first region has a maximum decomposition rate of $183^{\circ} \mathrm{C}$ with a minimum weight loss (2.6 wt \%). The second region has a maximum decomposition rate of $274^{\circ} \mathrm{C}$ with a weight loss of 5.7 wt $\%$. The third region in which the main thermal decomposition is produced is characterized by a complex multistep process between 300 and $450^{\circ} \mathrm{C}$, with maximum decomposition rates at 381 and $410^{\circ} \mathrm{C}$. 


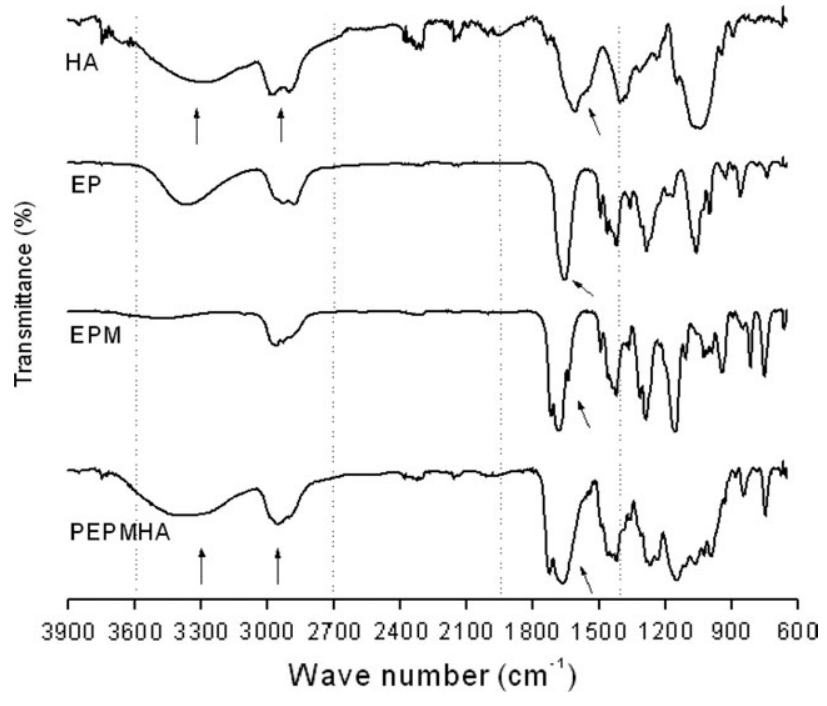

FIGURE 1. FTIR-ATR of HA, EP, EPM, and PEPM semi-interpenetrated with HA.

The first region of relatively low temperature corresponds to the loss of water bonded to HA and EPM polymeric chains by the cleavage of hydrogen bonds. This gives a clear idea of the retention of a low amount of water by the most polar and ionizable groups of both the polymeric components. The weight loss detected in a second region, with maximum decomposition rate at $274^{\circ} \mathrm{C}$, is probably due to the cleavage of PEPM-HA bonds in the semi-interpenetrated system and dehydration of glucuronic rings, in accordance to the values reported elsewhere. ${ }^{24}$ The third subsequent region of main degradation in the polymer can be characterized by possible modification reactions, through lateral chains that can originate new groups or crosslinking bonds, followed by a process of radical depolymerization, where the monomer is obtained as product of degradation. ${ }^{25}$ This degradation profile is more complicated than that observed for the homopolymer PEPM, hereby represented in a multistep behavior as a consequence of the strong interactions of the semi-interpenetrated system PEPMHA. ${ }^{26}$

\section{Morphologic characterization}

The morphology of freeze-dried PEPMHA systems was observed by SEM. Images of the sample surface are shown in Figure 3. Pore size varies from 7 to $12 \mu \mathrm{m}$ in the first series [Fig. 3(a,b); TEGDMA as crosslinker] and 12 to $30 \mu \mathrm{m}$ in the second series [Fig. 3(c,d); MBA as crosslinker]. The interconnected porous structure found can be related to the sample preparation procedure, and this is frozen at $-20^{\circ} \mathrm{C}$ and freeze dried. Freeze-drying techniques have widely shown their suitability for a range of hydrogel materials to produce interconnected porous architectures. ${ }^{27}$ However, this technique has also revealed difficulties in precisely tuning pore size as denoted in the heterogeneous porous structure observed in our results. Despite the influence of the freeze-drying process in the pores of the PEPMHA hydrogels, it can still be appreciated that the concentration of hyaluronic acid present in the hydrogels is affecting the overall porosity. It is also clear from the SEM images that hydrogels prepared with TEGDMA present higher interconnectivity than hydrogels prepared with MBA. Even though these images correspond to nonhydrated polymeric composite systems, the SEM technique has been used by other researchers to obtain a good manifestation of microstructural changes among different hydrogel samples. ${ }^{28}$

\section{Dynamic mechanical analysis}

To analyze the mechanical properties of the PEPMHA networks, hydrogels were subjected to consecutive frequency

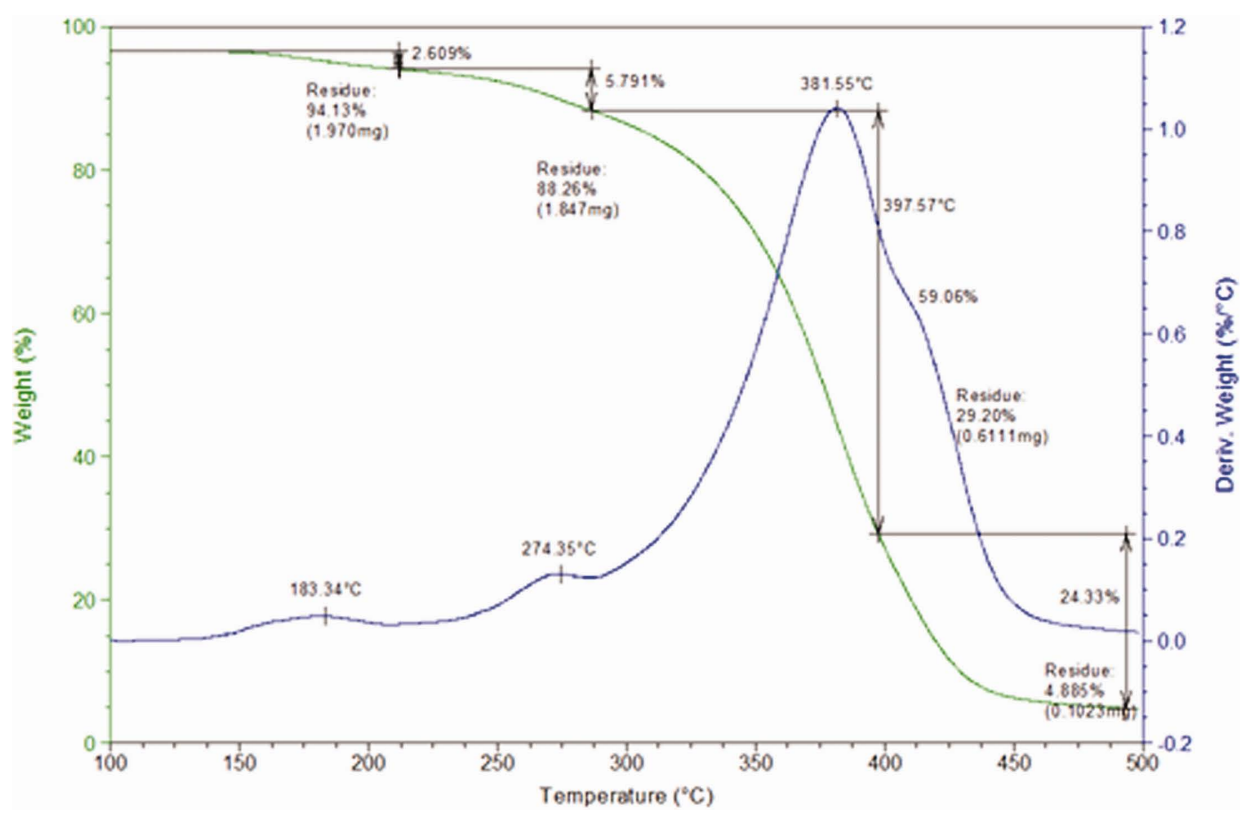

FIGURE 2. Thermogravimetric analysis of PEPMHA. [Color figure can be viewed in the online issue, which is available at wileyonlinelibrary.com.] 

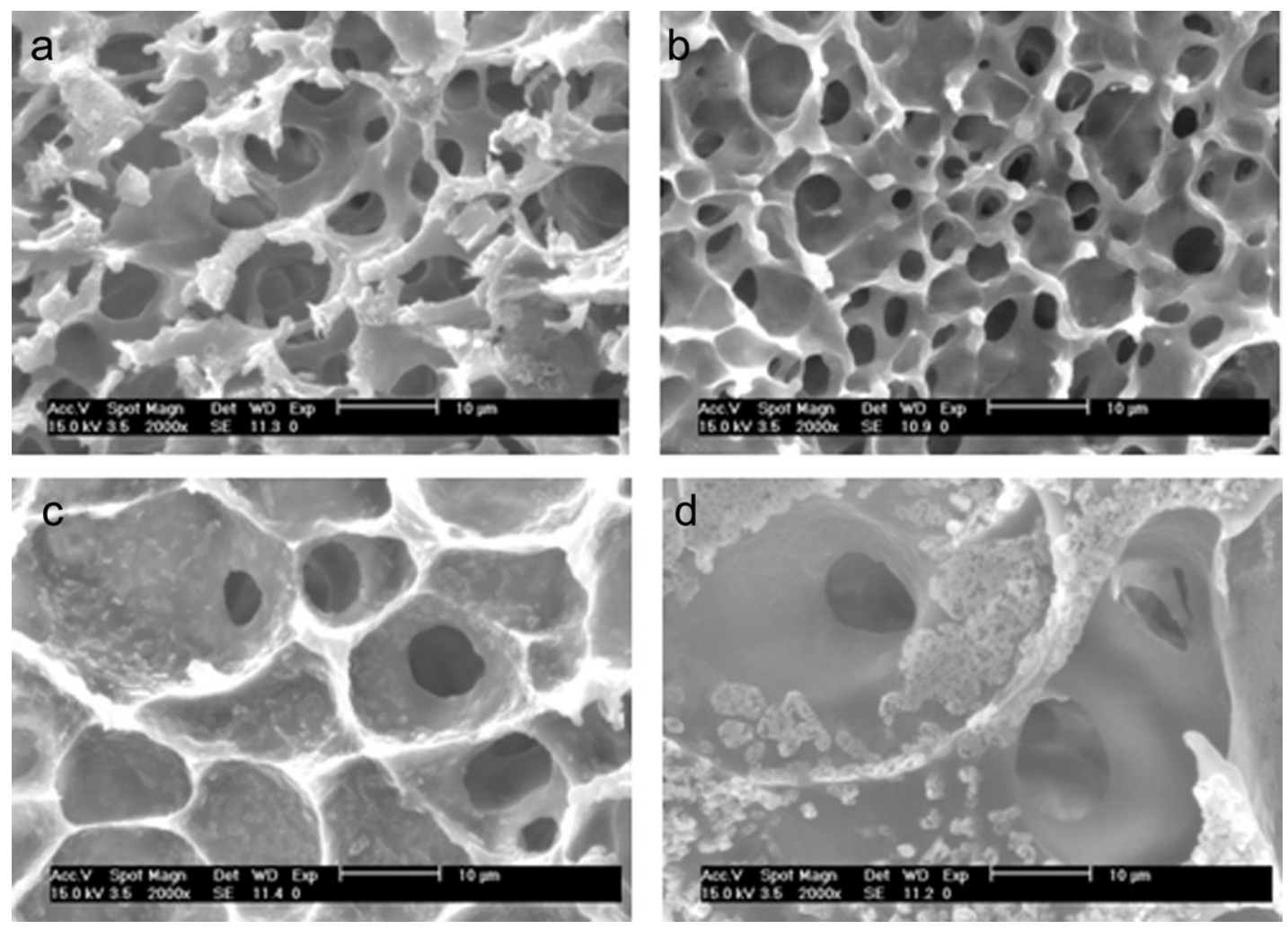

FIGURE 3. Scanning electron micrographs $(\times 2000)$ from EPM in the presence of HA $2.5 \%(a, c)$ or HA $5 \%(b, d)$, using potassium persulfate as initiator and triethylene glycol dimethacrylate (top) or $N, N^{\prime}$-methylenebisacrylamide (bottom) as crosslinker.

sweeps extending from 0.1 to $10 \mathrm{~Hz}$, at a temperature of $37^{\circ} \mathrm{C}$. Storage or elastic modulus $\left(G^{\prime}\right)$ and the loss tangent $(\tan \delta)$ were plotted as function of the frequency and both concentrations of HA and crosslinking agents [Fig. 4(a,b)]. From the obtained data, we can observe that the storage modulus of all hydrogels increased with increasing frequency, although the increased profiles were different. The modulus values of hydrogels prepared with TEGDMA increase at lower rates compared with those of hydrogels prepared with MBA. So, for the tested frequencies, the moduli increased from 0.452 to $1.3 \mathrm{MPa}$ and 0.19 to $0.69 \mathrm{MPa}$, for systems prepared with MBA (HA 2.5 and 5\%) and from 0.37 to $0.57 \mathrm{MPa}$ and 0.41 to $0.69 \mathrm{MPa}$ for systems prepared with TEGDMA (HA 2.5 and $5 \%$ ). Tan $\delta$ values (at $10 \mathrm{~Hz}$ ) ranged from 0.41 to 0.64 for systems prepared with MBA (HA 2.5, 5\%) and 0.24 to 0.29 for systems prepared with TEGDMA (HA 2.5, 5\%).

\section{Swelling studies}

The swelling pattern of PEPMHA hydrogels was followed periodically for 720 hours and is shown in Figure 5. After immersion in a phosphate buffer saline solution, at $37^{\circ} \mathrm{C}$, PEPMHA hydrogels readily swelled up to $350 \%$ of their initial weight, which suggest their highly hydrophilic character. All samples reached an equilibrium swelling state after 2.5 hours $(\% \mathrm{~S} \approx$ $266,244,235)$ except for PEPMHA hydrogels prepared with TEGDMA and HA 5\% that reached an apparent plateau within 1 hour ( $\% \mathrm{~S} \approx 328$ ). Furthermore, both samples prepared with TEGDMA have shown the capacity to absorb larger quan- tities of PBS. Swelling ratios increased with increased HA concentration, indicating a controlled hydrophilic balance in response to the strong ionic character of HA.

\section{Stimulus sensitivity of PEMHA hydrogels}

Figures 6 and 7 illustrate the effect of temperature $\left(20-50^{\circ} \mathrm{C}\right)$ on the equilibrium-swelling ratio of the different hydrogels. For the three different $\mathrm{pH}(2,7.4$, and 10$)$ settings, as the temperature increases, the swelling ratios of all samples increase. It was observed that at an acidic environment ( $\mathrm{pH} 2$ ), the temperature variation had a pronounced effect on the swelling profiles of hydrogels compared with the neutral $(\mathrm{pH} 7.4)$ or alkaline ( $\mathrm{pH} \mathrm{10)}$ media, which was a common feature for both MBA and TEGDMA hydrogels, but is more evident with regard to HA concentration (5\%). On the other hand, the data in both Figures 6 and 7 do not suggest a clear hydration-dehydration shift in response to temperature stimulation, as expected according to the thermoresponsive nature of PEPM hydrogels. Considering the $\mathrm{pH}$ variation, maximum swelling ratios were obtained at $\mathrm{pH} 10$ which was also verified when hydrogels were incubated at the same set of pHs and stabilized temperature, $20^{\circ} \mathrm{C}$ and $37^{\circ} \mathrm{C}$ baths, (data not shown). According to these results, it seems that the effect of the ionic strength of HA and its' interaction with PEPM chains interferes with the thermosensitivity of the PEPM homopolymer, in such a way that the observed responses could be considered as an average of both the thermosensitivity and the ionic strength associated with both constituents of the proposed sIPNs. 

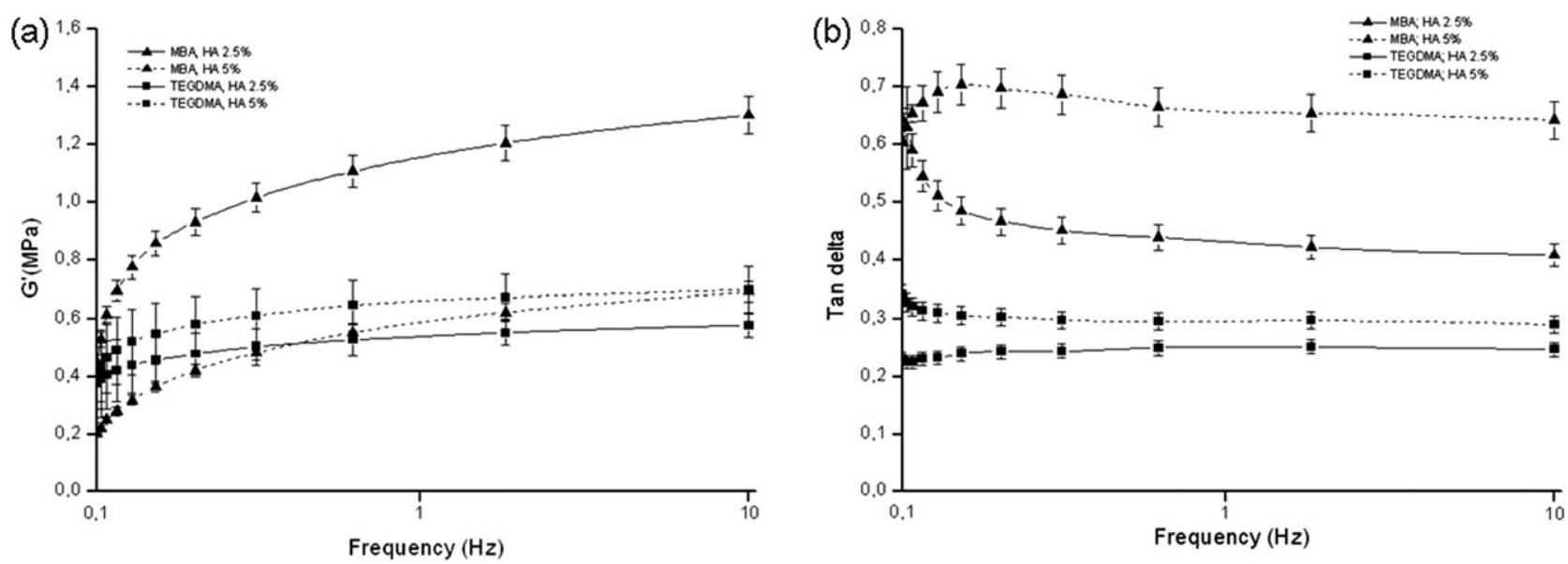

FIGURE 4. (a) Storage modulus $\left(G^{\prime}\right)$ and (b) loss tangent ( $\tan \delta$ ) of PEPMHA hydrogels according to the applied frequency measured at $37^{\circ} \mathrm{C}$, in a hydrated environment.

\section{Cell culture}

The cellular proliferative activity was quantitatively measured by the alamar blue assay after 48 hours of culture. The assay reveals the metabolic activity of cells, shown in Figure 8. All values of the samples are expressed as a percentage of the average control value. Cell proliferation on PEPMHA prepared with MBA or TEGDMA was found to be significantly increased compared with cells cultured on tissue culture polystyrene (TCPS, positive control), although they were not statistically different between them $(p>0.05)$. H\&E staining (micrograph in Fig. 8) supports efficient cellular adhesion and proliferation on the PEPMHA hydrogels.

\section{DISCUSSION}

Environment-sensitive PEPM hydrogel systems have evinced interest as drug delivery vehicles and have been considered recently for TE applications. ${ }^{29,30}$ These materials possess thermodynamically active functional groups on their polymer chain, making them sensitive to stimuli, a property that provides these gels with advantages over ordinary gels. ${ }^{31-33}$ The synthetic, hydrolytically degradable PEPM semi-interpenetrated with the unmodified, enzymatically degradable ECM component, HA, proposed in this study, can thus be an efficient approach to achieve new hybrid materials. One of the main challenges with hydrogels concerns the attainment of an optimal mechanical stability while maintaining their hydrophilic character. Hydrogels containing a high water level ( $>95 \%$ wt) usually have a compressive strength lower than $0.01 \mathrm{~Pa}$, which can be an obstacle in tissue regeneration. ${ }^{34}$ In our case, the concentration of HA was kept moderate in order to minimize the effects on the physical properties of the network. Still, incorporation of HA is expected to improve the network properties, considering its high water retention character, and enhance the biocompatible profile for biological applications.

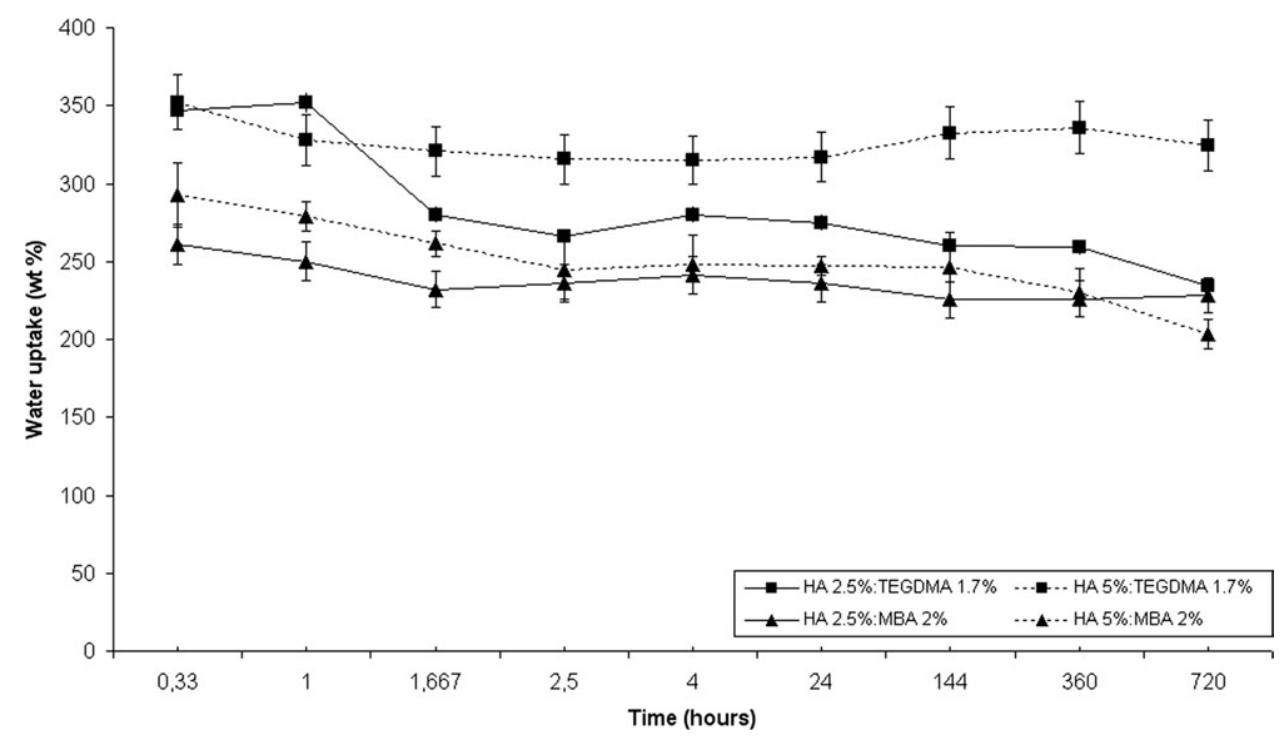

FIGURE 5. Swelling ratios are plotted as function of time. Hydrogels were incubated in a phosphate buffer solution of $\mathrm{pH} 7.4$, at $37^{\circ} \mathrm{C}$. 


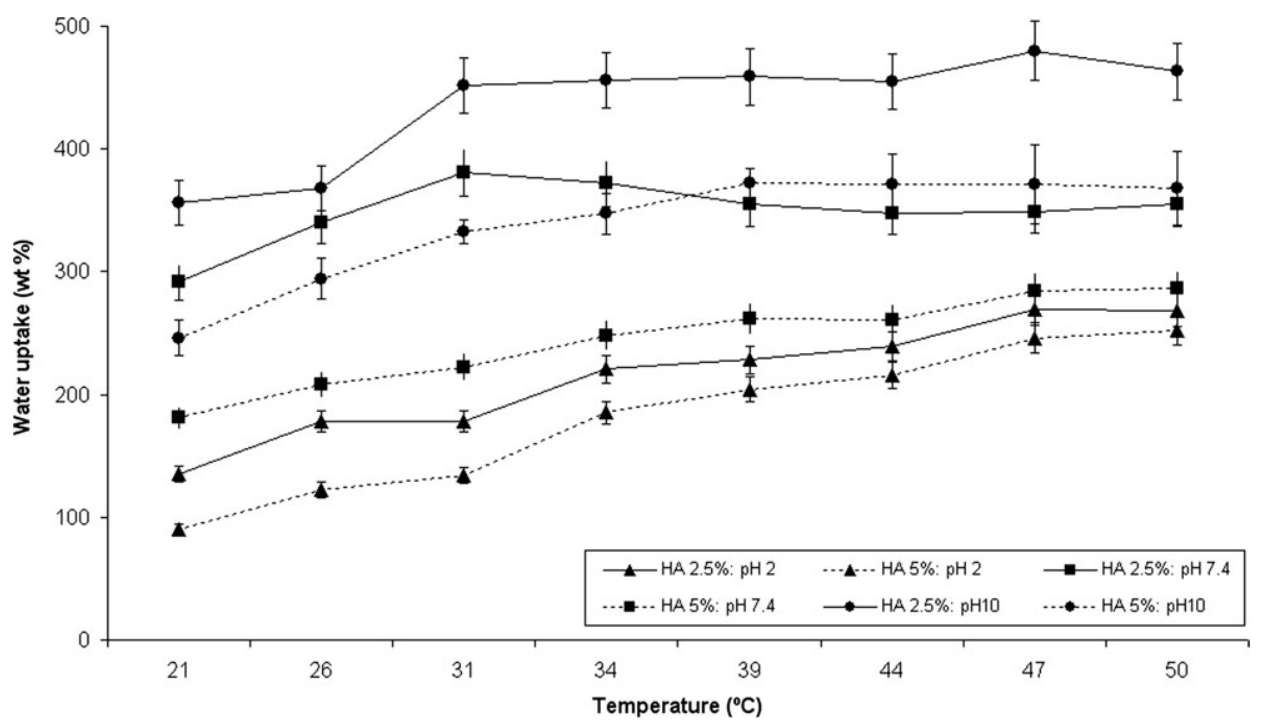

FIGURE 6. Temperature-dependent swelling kinetics of PEPMHA hydrogels (EPM $0.725 \mathrm{M}$, MBA $2 \%$, HA 2.5 and $5 \%$ ) in aqueous phosphate solutions at $\mathrm{pH} 2,7.4$, and 10.

Hereby, we have described the synthesis of PEPMHA hydrogels based on free-radical polymerization using two different crosslinkers and different concentrations of HA followed by freeze drying, also known as lyophilization, and which has been extensively used for the fabrication of porous hydrogels based on natural and synthetic hydrophilic polymers. ${ }^{27}$ FTIR-ATR characterization confirmed the successful synthesis of the systems. The main characteristic absorption bands were characterized and allowed us to attribute the PEPM and HA interactions to the peak corresponding to the $\mathrm{C}=0$ vibrations when compared with EPM and $\mathrm{HA}$ alone. From the thermal analysis, increased thermal stability was observed up to temperatures higher than the physiological temperature, which suggests that these systems when implanted will be able to maintain their thermal characteristics. Morphologic analysis revealed different porous and interconnected structures, which may render oxygen and nutrient exchange as well as offer space for cell adhesion and proliferation. As PEPMHA hydrogels are intended to be used in a hydrated environment, it is of relevance to predict their biomechanical behavior by testing the mechanical properties in simulated physiologic conditions, using DMA analysis. PEPMHA hydrogels have shown a different behavior depending on the crosslinking agent or the concentration of the hyaluronic acid used. The observed increase of the storage moduli with increasing frequency was more evident for systems prepared with MBA, revealing the highest modulus value (about twofold, at $10 \mathrm{~Hz}$ ) of the four groups, when the concentration of HA was 2.5\% [Fig. 4(a)]. This frequency dependency has been reported in

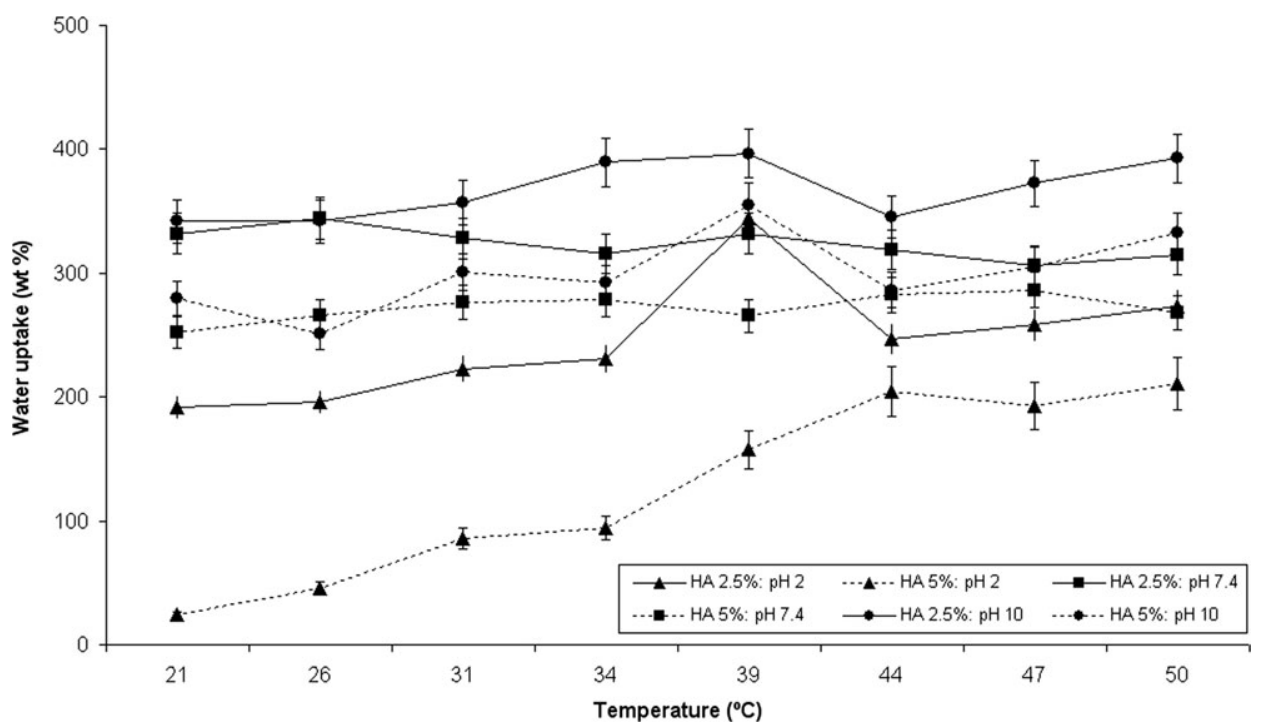

FIGURE 7. Temperature-dependent swelling kinetics of PEPMHA hydrogels (EPM $0.725 \mathrm{M}$, TEGDMA $1.7 \%$, HA 2.5 and $5 \%$ ) in aqueous phosphate solutions at $\mathrm{pH} 2,7.4$, and 10 . 


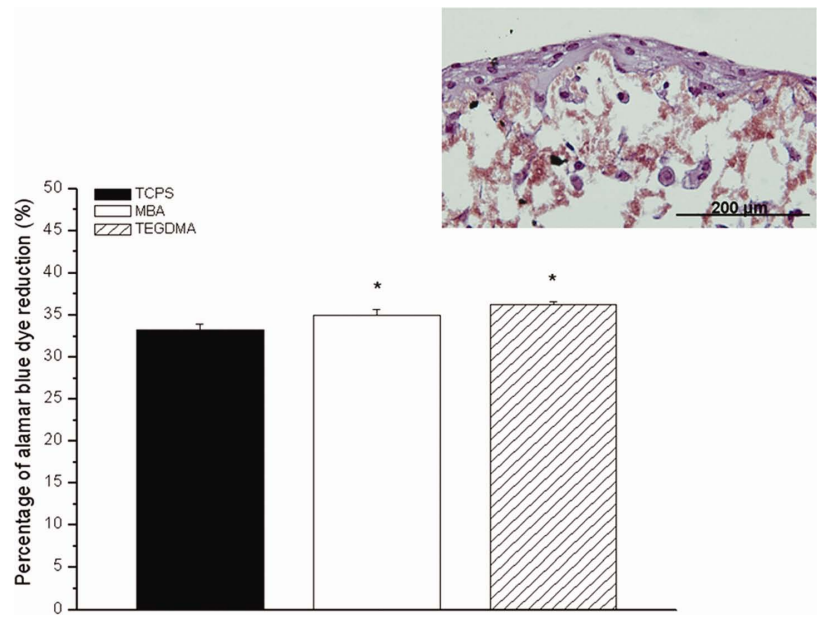

FIGURE 8. Alamar blue dye reduction assay after $48 \mathrm{~h}$ for PEPMHA (MBA or TEGDMA as crosslinker), with HA 5\% and the non-material control (cell-only). H\&E staining of BM-MSC cultured for $48 \mathrm{~h}$ on a PEPMHA (TEGDMA) hydrogel showed cell attachment and proliferation. [Color figure can be viewed in the online issue, which is available at wileyonlinelibrary.com.]

hydrogels and reflects the more difficult reaction of the conformational mobility of the chains for shorter variations of the periodical load. ${ }^{35}$ However, for systems prepared with TEGDMA, the elastic moduli were found to be almost parallel to each other (HA 2.5 and 5\%) and barely frequency dependent. This behavior has been observed for many biological gels in which the chains are physically crosslinked into a network. ${ }^{21}$ In addition, for these hydrogels, $G^{\prime}$ improved with increasing concentration of HA. This suggests that the presence of hyaluronic acid induces a reinforcement of the SIPNs. Xin and collaborators have also reported similar features in sIPNs, using LMW HA that interacts strongly with collagen due to the higher mobility of the chains and weaker interactions, acting as mechanical support. ${ }^{21}$ An opposite effect of HA concentration was observed for PEPMHA systems prepared with MBA. In this case, augmenting HA concentration (MBA, HA 5\%) could lead to an increase in prepolymer solution viscosity, which probably interferes in gel formation and affects the network mechanical stability, thus decreasing $G^{\prime}$ value.

The loss factor is the ratio of the amount of energy dissipated as heat by viscous mechanisms relative to the energy stored in the sample's elastic component. By comparing the loss tangent data of the four hydrogels, it is found that the viscous component values increased with increasing concentration of hyaluronic acid, which suggests the existence of an entangled network. Taking into account the damping property of each hydrogel, major differences were not found between hydrogels prepared with TEGDMA, evidencing that these systems present no frequency-dependent behavior. Hence, the contribution of both elastic and viscous components remains constant and therefore would be an advantage for the application for engineering musculoskeletal tissues. ${ }^{36}$ On the other hand, when we consider hydrogels prepared with MBA, these present opposite behaviors between them. Although for PEPMHA systems prepared with MBA (HA 2.5\%), the loss tangent decreases with increasing frequency, indicating a lower contribution of the elastic component, for a higher concentration of $\mathrm{HA}$ (5\%), the loss tangent increases at low frequencies and stabilizes at higher frequencies, similar to other hydrogels (TEGDMA). This behavior may render MBA-prepared PEPMHA hydrogels with weaker mechanical stability. Overall, these results highlight the role of specific interactions that occur between the PEPM-HA-crosslinkers during gel formation in controlling the network mechanical stability.

The distinct mechanical properties of the developed hydrogels can be associated with differences in the porosity and swelling behavior for each group. It is well known that the mechanical properties can be intimately related to the polymers' structure, crosslinking density, and swelling degree and therefore can be modulated through variations in the polymeric composition, crosslinking degree, and/or agents as well as the polymerization conditions. ${ }^{37}$ The effective amount of MBA for hydrogels' formation was superior to the amount of TEGDMA, and hence, at a higher crosslinking density the network chains have relatively less movement, thus originating more compact sIPNs, which may be supported by the relatively lower porosity and interconnectivity found when MBA was used as a crosslinker (Fig. 3). This fact could explain both the highest modulus value obtained (MBA, HA $2.5 \%$ ) and the lowest swelling ratio found (Fig. 5). ${ }^{38}$

The high water retention character of physically entangled HA was clearly visible in the PEPMHA composite systems. For the four sIPNs systems, when the amount of HA used is increased, there is a substantial increase in the swelling ratio, with the most hydrated hydrogels being the ones that were crosslinked with TEGDMA while using HA at 5\% (Fig. 5). This denotes the synergistic effect of HA, and that this molecule enhances the bonds with the surrounding water molecules, as predicted initially in the study, which is consistent with the results found for similar sIPNs fabricated with polymers of synthetic origin in the presence of different ECM materials. ${ }^{9}$ This effect is also supported by the improvement of the viscoelastic behavior [Fig. 4(b)] of the hydrogels prepared with HA 5\% in comparison with HA $2.5 \%$. Nonetheless, $\tan \delta$ values for hydrogels prepared with TEGDMA did not seem to correlate with the more hydrated structures.

Another potential mechanism reported in this paper is the sensitive response to variations in temperature and $\mathrm{pH}$. PEPM polymers were shown to have a negative sensitivity with temperature in aqueous solutions. ${ }^{26}$ According to various studies, hydrogels prepared by crosslinking thermosensitive polymers present a variation in their hydration degree, which is dependent on the temperature being below or above their critical transition temperature. This phenomenon is influenced by variations of molecular interactions in response to temperature. ${ }^{39}$ These types of polymers present both hydrophilic and hydrophobic groups, thus presenting an amphiphilic character (Scheme 1). It is the presence of these groups and the strength of the molecular interactions that will command the permeability of the water and the salts when the temperature is altered. 


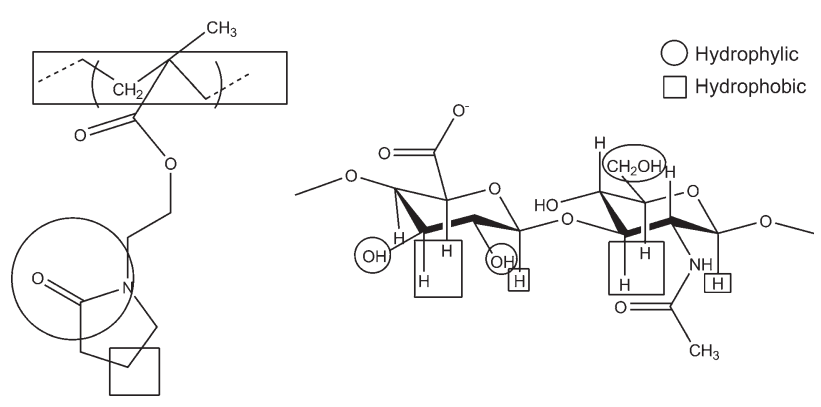

SCHEME 1. Structure of PEPM and HA and their respective hydrophilic and hydrophobic groups.

PEPM, due to its hydrophilic groups, as the cyclic amide in the pyrrolidone, can create hydrogen bonds with the water molecules and presents a critical transition temperature associated to a lower critical solution temperature (LCST) of $34^{\circ} \mathrm{C}$, meaning that when the temperature is below this value the polymer becomes highly soluble. ${ }^{40}$ Although in comparison with other methacrylate-based polymer, poly( $N$-acryloyl pyrrolidone), with LCST of $56^{\circ} \mathrm{C}$, presents a lower transition temperature due to the presence of hydrophobic groups. ${ }^{41}$ So, it would be expected that with the crosslinking of the EPM in the presence of a highly hydrophilic biopolymer, HA, the transition temperature of the polymeric system would be increased. But, in fact, the behavior that could be associated to a phase transition temperature of the semi-interpenetrated hydrogels was hardly defined and although there was in fact a variation in the swelling ratios, this was not sufficient to prove the existence of two different states of the polymeric network, expanded and collapsed, when the temperature was either less than or more than the critical transition temperature determined for crosslinked PEPM. ${ }^{26}$ It is worth noting that in other studies the swelling ratios for the sIPNs hydrogels were found to be almost independent of temperature. ${ }^{42,43}$ Another hypothesis for these findings is that probably the bonds of water-carboxylic groups of the hyaluronic acid molecule control the swelling behavior and mismatch the thermoresponsive character of the PEPM. Similar features have been observed in other polymeric systems. ${ }^{44}$

The incorporation of hyaluronic acid into the bulk formulation-induced pronounced $\mathrm{pH}$ sensitivity; the swelling ratio of the hydrogels was found to vary from $\approx 200 \%$, at acidic $\mathrm{pH}$, to $300-400 \%$, in physiological conditions. Regarding the nature of this biopolymer, presenting sensitivity to external stimulus, as variations in the $\mathrm{pH}$, when the carboxylic acid groups of the glucuronic acid residues are below the $\mathrm{p} K_{\mathrm{a}}(3-4)$, they are in the $-\mathrm{COOH}$ form and the low swelling values can be attributed to the presence of nonionic hydrophilic $\mathrm{COOH}$ and $-\mathrm{OH}$ groups in the hydrogel network. $^{45,46}$ As the $\mathrm{pH}$ of the solution increases, the $-\mathrm{COOH}$ groups become ionized to $\mathrm{COO}-$, and the electrostatic repulsion between the charges causes an enhancement in the swelling capacity. At $\mathrm{pH} \mathrm{7,} \mathrm{these} \mathrm{groups} \mathrm{are}$ predominantly ionized, due to the HA-associated exchangeable cation counterions that maintain charge neutrality. So, the $\mathrm{pH}$ sensitivity could be explained by the ionizable char- acter of hyaluronic acid and the conformation this polyanion molecule acquires in the presence of solutions with different acidity. Similar swelling $\mathrm{pH}$ dependencies have been reported. $^{47}$

Most of the strategies that use hyaluronic acid have been based on chemical modifications. ${ }^{48}$ However, this feature has been recognized to negatively influence on the cell's recognition, and therefore its biological capabilities are reduced. This was one of the reasons that in this work HA molecule has been maintained unmodified. Cellular studies showed an increase in the proliferation values of BM-MSCs when compared with cells grown on tissue-cultured polystyrene reference wells. The results also demonstrated cell spreading and proliferation when cultured in vitro, highlighting the biocompatible potential of these hydrogels.

\section{CONCLUSION}

Highly flexible and stable hydrogels based on semi-interpenetrating polymer networks could be easily prepared by free radical polymerization of EPM, at low or moderate temperature, in the presence of a natural component, hyaluronic acid, followed by lyophilization. The incorporation of HA to form sIPNs with the synthetic and hydrolytically degradable EPM improved the swelling capacity of these hydrogels and in some cases induced a mechanical reinforcement of the hydrogels. When the nontoxic crosslinking agent, TEGDMA, was used in the synthesis process, hydrogels achieved maximum swelling ratios and presented stable viscoelastic behavior. We found that the phase transition temperature of PEPMHA hydrogels was affected by the physically entangled hyaluronic acid that eventually led to loss of temperature sensitivity. In fact, the incorporation of HA into the structure induced pronounced $\mathrm{pH}$ sensitivity, so that the swelling of the hydrogels was seen to markedly vary over a range of $\mathrm{pH}$. This was mainly attributed to the HA ionizable character, in addition to the amphiphilic character of the PEPM. The biocompatible and noncytotoxic profile of PEPMHA hydrogels was demonstrated using BM-MSCs. Further in vitro studies are being performed in order to fully examine their biological performance. Additionally, this study opens news directions in the use of PEPMHA materials as drug delivery vehicles concerning $\mathrm{pH}$ internal body variations.

\section{ACKNOWLEDGMENTS}

The authors thank David Gomez, Sofia Caridade, and Justyna Chojnacka for their technical support and BIOIBERICA for the supply of hyaluronic acid. This work was carried out under the scope of the European NoE EXPERTISSUES, projects MAT 2007-63355, PTDC/QUI/68804/2006 (FCT), CIBER-BBN and Plan-E 2009-0144. In the memory of Prof. Roberto Sastre.

\section{REFERENCES}

1. Spiller KL, Laurencin SJ, Charlton D, Maher SA, Lowman AM. Superporous hydrogels for cartilage repair: Evaluation of the morphological and mechanical properties. Acta Biomater 2008;4: 17-25.

2. Klouda L, Mikos AG. Thermoresponsive hydrogels in biomedical applications. Eur J Pharm Biopharm 2008;68:34-45. 
3. Ruel-Gariépy $E$, Leroux J-C. In situ-forming hydrogels-review of temperature-sensitive systems. Eur J Pharmaceut Biopharmaceut 2004;58:409.

4. Drury JL, Mooney DJ. Hydrogels for tissue engineering: Scaffold design variables and applications. Biomaterials 2003;24:4337-4351.

5. Spiller KL, Maher SA, Lowman AM. Hydrogels for the repair of articular cartilage defects. Tissue Eng Part B Rev 2011;17:281-299.

6. Huebsch N, Mooney DJ. Inspiration and application in the evolution of biomaterials. Nature 2009;462:426-432.

7. Yan C, Pochan DJ. Rheological properties of peptide-based hydrogels for biomedical and other applications. Chem Soc Rev 2010; 39:3528-3540.

8. Chen J, Park H, Park K. Synthesis of superporous hydrogels: Hydrogels with fast swelling and superabsorbent properties. J Biomed Mater Res 1999;44:53-62.

9. Kutty JK, Cho E, Soo Lee J, Vyavahare NR, Webb K. The effect of hyaluronic acid incorporation on fibroblast spreading and proliferation within PEG-diacrylate based semi-interpenetrating networks. Biomaterials 2007;28:4928-4938.

10. Barnes CP, Sell SA, Boland ED, Simpson DG, Bowlin GL. Nanofiber technology: Designing the next generation of tissue engineering scaffolds. Adv Drug Deliv Rev 2007;59:1413-1433.

11. Kim MS, Jun I, Shin YM, Jang W, Kim SI, Shin H. The development of genipin-crosslinked poly(caprolactone) (PCL)/gelatin nanofibers for tissue engineering applications. Macromol Biosci 2010;10:91-100.

12. Vendamme R, Onoue SY, Nakao A, Kunitake T. Robust free-standing nanomembranes of organic/inorganic interpenetrating networks. Nat Mater 2006;5:494.

13. Peppas NA, Bures $P$, Leobandung W, Ichikawa $H$. Hydrogels in pharmaceutical formulations. Eur J Pharm Biopharm 2000;50: 27-46.

14. Langer R, Vacanti JP. Tissue engineering. Science 1993;260: 920-926.

15. González N, Elvira C, San Roman J. Hydrophilic and hydrophobic copolymer systems based on acrylic derivatives of pyrrolidone and pyrrolidine. J Polym Sci A Polym Chem 2003;41:395-407.

16. Gemeinhart RA, Chen J, Park H, Park K. pH-sensitivity of fast responsive superporous hydrogels. J Biomater Sci Polymer Ed 2000;11:1371-1380.

17. Mequanint K, Patel A, Bezuidenhout D. Synthesis, swelling behavior, and biocompatibility of novel physically cross-linked polyurethane-block-poly(glycerol methacylate) hydrogels. Biomacromolecules 2006;7:883-891.

18. Volpi N, Schiller J, Stern R, Soltés L. Role, metabolism, chemical modifications and applications of hyaluronan. Curr Med Chem 2009;16:1718-1745.

19. Barbucci $R$, Torricelli $P$, Fini $M$, Pasqui D, Favia $P$, Sardella E, d'Agostino R, Giardino R. Proliferative and re-defferentiative effects of photo-immobilized micro-patterned hyaluronan surfaces on chondrocyte cells. Biomaterials 2005;26:7596-7605.

20. Allison DD, Grande-Allen KJ. Review. Hyaluronan: A powerful tissue engineering tool. Tissue Eng 2006;12:2131-2140.

21. Xin X, Borzacchiello A, Netti PA, Ambrosio L, Nicolais L. Hyaluronic-acid-based semi-interpenetrating materials. J Biomater Sci Polymer Ed 2004;15:1223-1236.

22. Cuggino JC, Igarzabal CIA, Rueda JC, Quinzani LM, Komber H, Strumia MC. Synthesis and characterization of new hydrogels through copolymerization of $\mathrm{N}$-acryloyl-tris-(hydroxymethyl) aminomethane and different crosslinkers. Eur Polym J 2008;44: 3548-3555.

23. Coimbra $P$, Alves $P$, Valente $T A$, Santos $R$, Correia IJ, Ferreira $P$ Sodium hyaluronate/chitosan polyelectrolyte complex scaffolds for dental pulp regeneration: synthesis and characterization. Int $J$ Biol Macromol 2011;49:573-579.

24. Milella E, Brescia E, Massaro C, Ramires PA, Miglietta MR, Fiori $\mathrm{V}$, Aversa P. Physico-chemical properties and degradability of non-woven hyaluronan benzylic esters as tissue engineering scaffolds. Biomaterials 2002;23:1053-1063.

25. Moharram MA, Khafagi MG. Thermal behavior of poly(acrylic acid)-poly(vinyl pyrrolidone) and poly(acrylic acid)-metal-poly(vinyl pyrrolidone) complexes. J Appl Polym Sci 2006;102: 4049-4057.
26. Gonzalez N. Nuevos Sistemas Polimericos Sensibles a pH y Temperatura. Aplicaciones Biomedicas. Madrid: Complutense; 2006.

27. Annabi N, Nichol JW, Zhong X, Ji C, Koshy S, Khademhosseini A, Dehghani F. Controlling the porosity and microarchitecture of hydrogels for tissue engineering. Tissue Eng Part B Rev 2010;16: 371-383.

28. Suri S, Schmidt CE. Photopatterned collagen-hyaluronic acid interpenetrating polymer network hydrogels. Acta Biomater 2009; 5:2385-2397.

29. Gimeno MJ, Garcia-Esteo F, Garcia-Honduvilla N, Bellón JM, Buján J, San Román J. Polymer controlled drug delivery system for growth hormone. Drug Deliv J Deliv Target Therapeut Agent 2002;9:223-237.

30. Reis RL, San Román J. Biodegradable Systems in Tissue Engineering and Regenerative Medicine: Boca Raton, FL: CRC Press; 2004. 592 p.

31. Qiu Y, Park K. Environment-sensitive hydrogels for drug delivery. Adv Drug Deliv Rev 2001;53:321-339.

32. Zhang J, Yuan K, Wang YP, Zhang ST, Zhang J. Preparation and $\mathrm{pH}$ responsive behavior of poly(vinyl)alcohol-chitosan-poly(acrylic) acid full ipn hydrogels. J Bioact Compat Polym 2007;22:207-218.

33. Lee SC, Cho YW, Park K. Control of thermogelation properties of hydrophobically modified methylcellulose. J Bioact Compat Polym 2005;20:5-13.

34. Vinatier C, Guicheux J, Daculsi G, Layrolle P, Weiss P. Cartilage and bone tissue engineering using hydrogels. Biomed Mater Eng 2006;16:107-113.

35. Oliveira MB, Song W, Martín L, Oliveira SM, Caridade SG, Alonso M, Rodriguez-Cabello JC, Mano JF. Development of an injectable system based on elastin-like recombinamer particles for tissue engineering applications. Soft Matter 2011;7:6426-6434.

36. Yan LP, Oliveira JM, Oliveira AL, Caridade SG, Mano JF, Reis RL. Macro/microporous silk fibroin scaffolds with potential for articular cartilage and meniscus tissue engineering applications. Acta Biomater 2012;8:289-301.

37. Anseth KS, Bowman N, Peppas LB. Mechanical properties of hydrogels and their experimental determination. Biomaterials 1996;17:1647-1657.

38. Caridade SG, da Silva RMP, Reis RL, Mano JF. Effect of solvent-dependent viscoelastic properties of chitosan membranes on the permeation of 2-phenylethanol. Carbohydrate Polymers 2009;75:651-659.

39. Aguilar MR, Elvira C, Gallardo A, Vázquez B, San Román J. Smart polymers and their application as biomaterials. In: Topics in Tissue Engineering. Ashammakhi N, Reis RL, Chiellini E, editors. Volume 3; 2007, chapter 6.

40. Prabaharan M, Mano JF. Stimuli-responsive hydrogels based on polysaccharides incorporated with thermo-responsive polymers as novel biomaterials. Macromol Biosci 2006;6:991-1008.

41. Garnier S, Laschewsky A. Non-ionic amphiphilic block copolymers by RAFT-polymerization and their self-organization. Colloid Polym Sci 2006;284:1243-1254.

42. Wang M, Qiang JC, Fang Y, Hu DD, Cui YL, Fu XG. Preparation and properties of chitosan-poly( $\mathrm{N}$-isopropylacrylamide) semi-IPN hydrogels. J Polym Sci Part A Polym Chem 2000;38:474-481.

43. Verestiuc L, Ivanov C, Barbu E, Tsibouklis J. Dual-stimuli-responsive hydrogels based on poly( $\mathrm{N}$-isopropylacrylamide)/chitosan semiinterpenetrating networks. Int J Pharmaceut 2004;269:185-194.

44. Gan LH, Gan YY, Deen GR. Poly(N-acryloyl-N'propylpiperazine): a new stimuli-responsive polymer. Macromolecules 2000;33:7893-7897.

45. Hascall VC, Laurent TC. Hyaluronan: structure and physical properties. Glycoforum Group. 1997, http://www.glycoforum.gr.jp/ science/hyaluronan.

46. Sadeghi M, Hosseinzadeh $\mathrm{H}$. Synthesis of starch-poly(sodium acrylate-co-acrylamide) superabsorbent hydrogel with salt and $\mathrm{pH}$ responsiveness properties as a drug delivery system. J Bioact Compat Polym 2008;23:381-404.

47. Kim SJ, Yoon SG, Lee YH, Kim SI. Bending behavior of hydrogels composed of poly(methacrylic) acid and alginate by electrical stimulus. Polym Int 2004:53:1456-1460.

48. Schanté CE, Zuber G, Herlin C, Vandamme TF. Chemical modifications of hyaluronic acid for the synthesis of derivatives for a broad range of biomedical applications. Carbohydr Polym 2011; 85:469-489. 